\section{Quantum Codes of Minimum Distance Two}

\author{
Eric M. Rains
}

\begin{abstract}
It is reasonable to expect the theory of quantum codes to be simplified in the case of codes of minimum distance 2 ; thus it makes sense to examine such codes in the hopes that techniques that prove effective there will generalize. With this in mind, we present a number of results on codes of minimum distance 2 . We first compute the linear programming bound on the dimension of such a code, then show that this bound can only be attained when the code either is of even length, or is of length 3 or 5 . We next consider questions of uniqueness, showing that the optimal code of length 2 or 4 is unique (implying that the well-known one-qubit-infive single-error correcting code is unique), and presenting nonadditive optimal codes of all greater even lengths. Finally, we compute the full automorphism group of the more important distance 2 codes, allowing us to determine the full automorphism group of any GF (4)-linear code.
\end{abstract}

Index Terms-Automorphisms, classification, quantum codes, uniqueness.

\section{INTRODUCTION}

In classical coding theory, the simplest nontrivial codes are the codes of minimum distance 2 , and their duals, repetition codes. Here, binary codes of minimum distance 2 are unique (consisting of all even-weight vectors), linear, and essentially trivial to analyze. In the quantum setting, as we shall see, the situation is not nearly so nice. The purpose of the present work is to explore the structure of quantum codes of minimum distance 2 , both because the theory is likely to be simpler in that case, and because many codes of interest (e.g., GF (4)-linear codes [1]) are built out of distance 2 codes.

Before proceeding with our study of distance 2 codes, it will be helpful to recall the concept of "additive" quantum codes [1]. These are constructed as follows: Given an additive (classical) code $C$ over GF (4), self-orthogonal under the inner product $\operatorname{Tr}(v \cdot \bar{w})$, apply the substitution

$$
0 \mapsto 1,1 \mapsto \sigma_{x}, \omega \mapsto \sigma_{y}, \bar{\omega} \mapsto \sigma_{z}
$$

to obtain a set of (commuting) operators. If the original code had dimension $k$ (over GF (2)), then these operators have $2^{k}$ joint eigenspaces, each of dimension $2^{n-k}$. Each of these eigenspaces is then an additive quantum code, of minimum distance given by the minimal weight of $C^{\perp}-C$; distinct eigenspaces give equivalent codes. For instance, the additive code with generator matrix

$$
\left[\begin{array}{cccc}
1 & 1 & 1 & 1 \\
\omega & \omega & \omega & \omega
\end{array}\right]
$$

gives rise to a quantum code of minimum distance 2 and dimension 4 , with an explicit basis of the form

$$
|0000\rangle+|1111\rangle,|0011\rangle+|1100\rangle,|0101\rangle+|1010\rangle,|0110\rangle+|1001\rangle \text {. }
$$

It is easy to verify that this is a joint eigenspace of

$$
\sigma_{x} \otimes \sigma_{x} \otimes \sigma_{x} \otimes \sigma_{x}
$$

and

$$
\sigma_{z} \otimes \sigma_{z} \otimes \sigma_{z} \otimes \sigma_{z}
$$

Manuscript received May 26, 1997; revised March 4, 1998.

The author is with AT\&T Research, Florham Park, NJ 07932-0971 USA. Communicated by D. Stinson, Associate Editor for Complexity and Cryptography.

Publisher Item Identifier S 0018-9448(99)00082-6.
We recall the notation that an $[[n, k, d]]$ refers to an additive code encoding $k$ qubits in $n$ qubits, with minimum distance $d$; an $((n, K, d))$ refers to a general code encoding $K$ states in $n$ qubits with minimum distance $d$. Thus the code $(1)$ is a $((4,4,2))$ and a $[[4,2,2]]$. Also, we will say that two codes are locally equivalent if there is an element of $P S U(2)^{\otimes n}$ mapping one into the other; we will say they are globally equivalent, or just equivalent, if it is possible to permute the qubits of one in such a way as to make it locally equivalent to the other. A code will be said to be nonadditive if it is not equivalent to any additive code.

The first natural question to ask about distance 2 codes is: How good can they be? That is, how large can the dimension of a distance 2 code be, given its length? In the case of even length $2 m$ this is easy; the quantum Singleton bound ([3]) states that the dimension can be at most $4^{m-1}$, while the additive code with generator matrix

$$
\left[\begin{array}{ccccc}
1 & 1 & 1 & \cdots & 1 \\
\omega & \omega & \omega & \cdots & \omega
\end{array}\right]
$$

attains this bound. For odd length, the situation is not as simple as this; however, one can use linear programming to give a bound. The resulting bound could, in principle, be met whenever the length is of the form $2^{m}+1$ (and is, indeed, met, when $m=2$ [8]); however, as we shall see, this does not happen. We also give a construction which, given a pure $((n, K, 2))$, produces a pure $((n+2,4 K, 2))$, thus giving a lower bound of $3 \cdot 2^{n-4}$ on the optimal dimension for $n$ odd.

Since the code (2), which we shall refer to as "the $[[2 n, 2(n-$ $1), 2]]$ " in the sequel, has a particularly nice structure, with a very large symmetry group, it is reasonable to conjecture that any optimal distance 2 code of even length is of that form. It is easy to see that this is, indeed, the case for length 2 ; on the other hand, we construct a nonadditive optimal code of length 6 , which allows the construction of nonadditive optimal codes of all larger even lengths. This leaves only length 4 open; by considering the quartic invariants of such a code [5], we show that the optimal length 4 code is unique as well. As the well-known $[[5,1,3]]$ and its associated self-dual $[[6,0,4]]$ can be built up from $((4,4,2))$ 's, we can show that those codes are unique as well.

Finally, we consider the question of the full automorphism group of the codes (2); that is, automorphisms that do not necessarily lie in the Clifford group. This question is important, for instance, because automorphisms of a code induce fault-tolerant operations [2]. Here, we find that such nonadditive automorphisms can occur only for length 2. Since GF (4)-linear codes are built out of [[2n, 2( $n-$ $1), 2]]$ 's, we find, for instance, that any equivalence between GF (4)linear codes (subject to certain trivial restrictions) must lie in the Clifford group.

A remark on notation: If $\rho$ is an operator on a tensor product Hilbert space $V_{1} \otimes V_{2} \otimes \cdots \otimes V_{n}$, and $S$ is any subset of $\{1,2, \cdots n\}$, then we define $\operatorname{Tr}_{S}(\rho)$ to be the partial trace of $\rho$ with respect to the spaces indexed by $S$; thus $\operatorname{Tr}_{S}(\rho)$ is an operator on $\bigotimes_{i \notin S} V_{i}$.

\section{BOUNDS}

We first recall the following fact.

Theorem 1: Let $Q$ be a $((2 m, K, 2))$ for some $m$ and $K$. Then $K \leq 4^{m-1}$. On the other hand, for all $m \geq 1$, there exists a $\left(\left(2 \bar{m}, 4^{m-1}, 2\right)\right)$.

Proof: The quantum Singleton bound ([3]; while Knill and Laflamme only consider the case $d$ odd, their proof easily extends to 
even $d[4]))$, states that for any $((n, K, d))$, we must have

$$
K \leq 2^{n-2(d-1)} \text {. }
$$

Applying this for $d=2$, we get the stated bound.

As remarked in Section I, existence follows from consideration of the code $(2)$

For odd length, the linear programming bound ([6, Theorem 10], see also [9]; note that the Singleton bound is a special case) is more complicated, but still feasible to compute:

Theorem 2: Let $Q$ be a $((2 m+1, K, 2))$ for some $m$ and $K$. Then

$$
K \leq 4^{m-1}\left(2-\frac{1}{m}\right) .
$$

Proof: We consider the coefficients $B_{0}, B_{1}$, and $S_{0}$ of the dual and shadow enumerators of $Q$. These can be expressed in terms of the weight enumerator of $Q$ as follows:

$$
\begin{aligned}
& B_{0}=2^{-n} \sum_{0 \leq i \leq n} A_{i} \\
& B_{1}=2^{-n} \sum_{0 \leq i \leq n}(3 n-4 i) A_{i} \\
& S_{0}=2^{-n} \sum_{0 \leq i \leq n}(-1)^{i} A_{i}
\end{aligned}
$$

where $n=2 m+1$. Eliminating $A_{n}$ and $A_{n-1}$ we get

$$
\begin{aligned}
(n-2) B_{0}+B_{1} & -2 S_{0} \\
& =2^{-n} \sum_{0 \leq i \leq m} 4(n-2 i-1)\left(A_{2 i}+A_{2 i+1}\right) .
\end{aligned}
$$

Now, since $Q$ has minimum distance 2, it follows that $K B_{0}=A_{0}$ and $K B_{1}=A_{1}$. Substituting in, we find

$$
\begin{aligned}
\left(K^{-1}(n-2)\right. & \left.-2^{-n}(4 n-4)\right) A_{0}+\left(K^{-1}-2^{-n}(4 n-4)\right) A_{1} \\
& =2 S_{0}+2^{-n} \sum_{1 \leq i<m} 4(n-2 i-1)\left(A_{2 i}+A_{2 i+1}\right) .
\end{aligned}
$$

The coefficients on the right-hand side are positive, while the coefficients on the left-hand side are negative whenever $K>4^{m-1}(2-$ $(1 / m))$. This contradicts the fact that $A_{0}>0$, while all other $A_{i}$ and $S_{0}$ are nonnegative.

It is straightforward to verifiy that the enumerator

$$
\begin{aligned}
A(x, y)= & \left(4^{m-1}\left(2-\frac{1}{m}\right)\right)^{2} \\
& \cdot\left(x^{n}+\frac{n}{n-2} x y^{n-1}+\frac{2 n-2}{n-2} y^{n}\right)
\end{aligned}
$$

satisfies all the constraints of [6, Theorem 10], with $K=4^{m-1}(2-$ $1 / \mathrm{m})$; thus this bound agrees with the full linear programming bound.

The bound (3) is integral precisely when $m$ is a power of 2 ; thus it is natural to conjecture that it can be met then. For $n=3$, the bound is $K \leq 1$, met, for instance, by the self-dual additive code

$$
\left[\begin{array}{lll}
1 & 1 & 0 \\
0 & 1 & 1 \\
\omega & \omega & \omega
\end{array}\right] .
$$

For $n=5$, the bound is $K \leq 6$, which is attained by the $((5,6,2))$ given in [8]. Thus it is somewhat surprising that these are the only cases in which the bound can be met.

Theorem 3: For no $i \geq 3$ is there a $\left(\left(2^{i}+1,2^{2^{i}-1}-2^{2^{i}-i-1}, 2\right)\right)$.
Proof: Suppose $Q$ were such a code. From the proof of Theorem 2, we can conclude that $S_{0}=0$, and $A_{i}=0$ for $1 \leq i \leq n-2$. Solving the equations $A_{0}=K^{2}, B_{0}=K$, and $K B_{1}=A_{1}$, we find that the weight enumerator of $Q$ must be

$$
\left(2^{2^{i}-1}-2^{2^{i}-i-1}\right)^{2}\left(x^{2^{i}+1}+\frac{2^{i}+1}{2^{i}-1} x y^{2^{i}}+\frac{2^{i+1}}{2^{i}-1} y^{2^{i}+1}\right) .
$$

Now, since $Q$ is pure of minimum distance 2 , if we trace away one qubit of $Q$, the resulting operator (e.g., $\operatorname{Tr}_{\{1\}}(Q)$ ) must be $P_{Q^{\prime}} / 2$ for some code $Q^{\prime}$. Partial tracing cannot introduce any new nonzero coefficients, so it follows that the weight enumerator of $Q^{\prime}$ must be of the form $a x^{2^{i}}+b y^{2^{i}}$. But $Q^{\prime}$ must have dimension $2^{2^{i}}-2^{2^{i}-i}$, and thus has weight enumerator

$$
\left(2^{2^{i}}-2^{2^{i}-i}\right)^{2}\left(x^{2^{i}}+\frac{1}{2^{i}-1} y^{2^{i}}\right) .
$$

Consider the orthogonal complement of $Q^{\prime}$. We can compute its weight enumerator, by noting that orthogonal complementation leaves each $A_{i}$ unchanged, except that it changes $A_{0}$ from $K^{2}$ to $\left(2^{n}-K\right)^{2}$. Thus the orthogonal complement of $Q^{\prime}$ has weight enumerator

$$
\left(2^{2^{i}-i}\right)^{2}\left(x^{2^{i}}+\left(2^{i}-1\right) y^{2^{i}}\right),
$$

and dual enumerator

$$
2^{2^{i}-i}\left(x^{2^{i}}+\left(4-2^{i}\right) x^{2^{i}-1} y+\cdots\right) .
$$

For $i>2,4-2^{i}$ is negative, giving a contradiction.

Remark: This result is quite fragile; in particular, it depends strongly on the fact that the code $Q$ must be pure. Consequently, the above argument cannot be used to strengthen the bound (3) except to say that it cannot be met. It should be possible to give a different proof of Theorem 3 that works for slightly suboptimal enumerators as well.

Remark: For $i=2$, the orthogonal complement of the code $Q^{\prime}$ has weight enumerator

$$
16 x^{4}+48 y^{4}
$$

which is the weight enumerator of a $((4,4,2))$; we will use this fact in the sequel.

The best lower bound we have been able to prove is the following:

Theorem 4: For all $m \geq 2$, there exists a pure $((2 m+1,3$. $\left.\left.2^{2 m-3}, 2\right)\right)$.

Proof: This was shown for $m=2$ in [8]. For $m>2$, we will need the following lemma:

Lemma 5: If there exists a pure $((n, K, 2))$, then there exists a pure $((n+2,4 K, 2))$.

Proof: Let $Q$ be a pure $((n, K, 2))$, and let $v_{2}$ be the state $|00\rangle+|11\rangle$ on two qubits. Then the new code $Q^{\prime}$ is the span of

$$
\begin{array}{r}
\left\{Q \otimes v_{2},\left(X_{1} \otimes X_{1}\right)\left(Q \otimes v_{2}\right),\left(Y_{1} \otimes Y_{1}\right)\left(Q \otimes v_{2}\right),\right. \\
\left.\left(Z_{1} \otimes Z_{1}\right)\left(Q \otimes v_{2}\right)\right\}
\end{array}
$$

where $X_{1}$ is the operator that acts as $\sigma_{x}$ on the first qubit and as the identity on the remaining qubits, and similarly for $Y_{1}$ and $Z_{1}$.

Examination of the appended pair of qubits tells us that $Q^{\prime}$ has four times the dimension of $Q$; it remains only to show that $Q^{\prime}$ is pure of minimum distance 2 . In other words, we need to show that any singlequbit error takes $Q^{\prime}$ to an orthogonal code; this is straightforward to verify.

The theorem follows by induction. 
Lemma 5 suggests the following natural question: What is the value of

$$
\kappa_{2}=\lim _{m \rightarrow \infty} 4^{1-m} K_{0}(2 m+1)
$$

where $K_{0}(2 m+1)$ is the maximum dimension of a pure $(2 m+$ $1, K, 2)$ ? By the lemma, this sequence is nondecreasing, so the limit exists, and is bounded between $(3 / 2)$ (by Theorem 4 ), and 2 (by Theorem 2).

\section{UNIQUENESS RESULTS}

It is natural to wonder whether the codes (1) are necessarily the only optimal distance 2 codes of even length. For length 2, the answer is an easy "Yes"; indeed, one can show the following stronger result:

Lemma 6: Let $w_{1}, w_{2}, w_{3}$, and $w_{4}$ be an orthonormal basis of $\mathbb{C}^{2} \otimes \mathbb{C}^{2}$ consisting of $((2,1,2))$ 's. Then there exists elements $U_{1}$ and $U_{2}$ of $S U(2)$ such that

$$
\begin{aligned}
& \left(U_{1} \otimes U_{2}\right) w_{1} \propto(|00\rangle+|11\rangle) / \sqrt{2} \\
& \left(U_{1} \otimes U_{2}\right) w_{2} \propto(|01\rangle+|10\rangle) / \sqrt{2} \\
& \left(U_{1} \otimes U_{2}\right) w_{3} \propto-i(|01\rangle-|10\rangle) / \sqrt{2} \\
& \left(U_{1} \otimes U_{2}\right) w_{4} \propto(|00\rangle-|11\rangle) / \sqrt{2} .
\end{aligned}
$$

Proof: Let $w=a|00\rangle+b|01\rangle+c|10\rangle+d|11\rangle$ have norm 1. For $w$ to have distance 2, we must have

$$
\operatorname{Tr}_{1}\left(w w^{\dagger}\right)=\operatorname{Tr}_{2}\left(w w^{\dagger}\right)=I / 2
$$

This gives the conditions

$$
\begin{aligned}
|a|^{2}+|c|^{2} & =1 / 2 \\
|b|^{2}+|d|^{2} & =1 / 2 \\
a \bar{b}+c \bar{d} & =0 .
\end{aligned}
$$

In other words, the matrix

$$
M(w)=\sqrt{2}\left(\begin{array}{ll}
a & b \\
c & d
\end{array}\right)
$$

must be unitary. Thus the theorem is equivalent to the statement that, for any 2 by 2 unitary matrices $M_{1}, M_{2}, M_{3}$, and $M_{4}$, orthonormal under the inner product $\frac{1}{2} \operatorname{Tr}\left(A B^{\dagger}\right)$, there exist unitary matrices $U_{1}$ and $U_{2}$ such that

$$
\begin{aligned}
& U_{1} M_{1} U_{2}^{t} \propto I \quad U_{1} M_{2} U_{2}^{t} \propto \sigma_{x}, \\
& U_{1} M_{3} U_{2}^{t} \propto \sigma_{y} \quad U_{1} M_{4} U_{2}^{t} \propto \sigma_{z} .
\end{aligned}
$$

To satisfy the first equation, we may take $U_{2}=\overline{U_{1} M_{1}}$; we may therefore assume $M_{1}=I$, without loss of generality. Then, up to phase, we have $M_{2}^{2}=M_{3}^{2}=M_{4}^{2}=I$. It follows that each of $M_{2}, M_{3}$, and $M_{4}$ can be written as real linear combinations of $\sigma_{x}, \sigma_{y}$, and $\sigma_{z}$; this determines an orthonormal basis of $\mathbb{R}^{3}$. Conjugation by $S U(2)$ acts as $S O(3)$ on $\mathbb{R}^{3}$; consequently, we may take the given basis to the standard basis. The resulting transformation gives us unitary matrices as desired.

Thus, in particular, any $((2,1,2))$ is locally equivalent to the $[[2,0,2]]$.

For $n \geq 6$, on the other hand, the answer is "No."

Theorem 7: For all even $m \geq 3$, there exists a nonadditive $\left(\left(2 m, 4^{m-1}, 2\right)\right)$.
Proof: In light of Lemma 5, it suffices to prove the result for $m=3$, which we may do by explicit construction. Let $v$ be the vector

$$
(|00\rangle+|11\rangle) \otimes(|00\rangle+|11\rangle) \otimes(|00\rangle+|11\rangle) .
$$

The new code will be the span of 16 translates of $v$ under the extraspecial group.

Consider the following nonlinear code of length 3 and minimum distance 2 over GF (4):

$$
\begin{aligned}
& \{000,011,0 \omega \omega, 0 \overline{\omega \omega}, \\
& 101,110,1 \omega \bar{\omega}, 1 \bar{\omega} \omega, \\
& \omega 0 \omega, \omega 1 \bar{\omega}, \omega \omega 1, \omega \bar{\omega} 0, \\
& \bar{\omega} 0 \bar{\omega}, \bar{\omega} 1 \omega, \bar{\omega} \omega 0, \overline{\omega \omega} 1\} .
\end{aligned}
$$

To this code, we can associate a set of operators from the extraspecial group, by mapping 0 to the identity, 1 to $Y_{1}, \omega$ to $X_{1}$, and $\bar{\omega}$ to $Z_{1}$. This, then, determines a set of translates of $v$, the span of which is easily verified to be a $((6,16,2))$. It remains only to show that this code is nonadditive; this can be done, for instance, by checking that some quartic invariant differs from that of the additive $[[6,4,2]]$.

Thus only length 4 remains open.

Theorem 8: Any $((4,4,2))$ is locally equivalent to the $[[4,2,2]]$.

Proof: Let $Q$ be a $((4,4,2))$. We proceed by first showing that for any pair of qubits of $Q$ there exists an orthonormal basis of the tensor product of those qubits such that the corresponding projection operators all commute with $P_{Q}$; moreover, it will turn out that, up to equivalence, the basis can be taken to be the cosets of the $[[2,0,2]]$. We will then consider the consistency conditions between the bases corresponding to different pairs of qubits; the result will follow.

From the proof of [5, Theorem 6] we know that for any $S \subset$ $\{1,2,3,4\}$ with $|S|=2$

$$
E_{v \in Q}\left(\operatorname{Tr}\left(-\left[\operatorname{Tr}_{S^{c}}\left(v v^{\dagger}\right) \otimes I, P_{Q}\right]^{2}\right)\right)=0 .
$$

In other words, for all $v \in Q, T_{S}(v) \otimes I$ commutes with $P_{Q}$, where we define

$$
T_{S}(v)=\operatorname{Tr}_{S c}\left(v v^{\dagger}\right) .
$$

Consequently, for each eigenspace of $T_{S}(v)$, the corresponding projection operator commutes with $P_{Q}$. If $T_{S}(v)$ has four distinct eigenvalues, we obtain a basis as desired. Otherwise, let $\Pi^{\prime}$ be the projection onto an eigenspace of $T_{S}(v)$. The operator $\Pi^{\prime} \otimes I$ commutes with $P_{Q}$, so the operator

$$
P_{Q}\left(\Pi^{\prime} \otimes I\right)
$$

is the projection onto a subcode $Q^{\prime}$ of $Q$. Take $v^{\prime} \in Q^{\prime}$ and consider $T_{S}\left(v^{\prime}\right)=\Pi^{\prime} T_{S}\left(v^{\prime}\right)$. If this separates $\Pi^{\prime}$, we get the desired basis by induction. Suppose, on the other hand, that for all $v^{\prime} \in Q^{\prime}, T_{S}\left(v^{\prime}\right) \propto \Pi^{\prime}$. But then the code $Q^{\prime}$ can correct for the erasure of both qubits in $S$. This is impossible, by the Singleton bound [3], unless $\operatorname{dim}\left(Q^{\prime}\right)=1$. But then, adding up the dimensions of the induced partition of $Q$, we conclude that we must have partitioned $Q$ into four subspaces, giving the desired basis.

Let $v_{1}, v_{2}, v_{3}$, and $v_{4}$ be the codewords of $Q$ corresponding to the orthonormal basis of $\mathbb{C}^{2} \otimes \mathbb{C}^{2}$ we have just constructed. Note that the projection onto each basis element is of the form

$$
\operatorname{Tr}_{S c}\left(v_{i} v_{i}^{\dagger}\right)
$$

for $i$ ranging from 1 to 4 . But then, if we trace away either remaining qubit, we get $I / 2$; it follows that each basis element is a $((2,1,2))$. 
Then Lemma 6 applies; consequently, we may assume without loss of generality that $P_{Q}$ commutes with the group

$$
G_{12}=\left\langle\sigma_{x} \otimes \sigma_{x} \otimes 1 \otimes 1, \sigma_{z} \otimes \sigma_{z} \otimes 1 \otimes 1\right\rangle
$$

by using an equivalence to map the orthonormal basis of $\mathbb{C}^{2} \otimes \mathbb{C}^{2}$ associated with $S=\{1,2\}$ to the cosets of the $[[2,0,2]]$.

Consider the basis associated to $S=\{1,3\}$. We are still free to transform the third qubit; this allows us to assert that $P_{Q}$ commutes with

$$
T_{x}=\left(a_{x x} \sigma_{x}+a_{y x} \sigma_{y}+a_{z x} \sigma_{z}\right) \otimes 1 \otimes \sigma_{x} \otimes 1
$$

with $\left|a_{x x}\right|^{2}+\left|a_{y x}\right|^{2}+\left|a_{z x}\right|^{2}=1$, and, similarly, for $T_{y}$ and $T_{z}$. On the other hand, $P_{Q}$ can be written as a linear combination of elements of the form

$$
e_{1} \otimes e_{1} \otimes e_{2} \otimes e_{3}
$$

where each $e_{i}$ is in $\left\{1, \sigma_{x}, \sigma_{y}, \sigma_{z}\right\}$; this follows from the assumption that $P_{Q}$ commutes with $G_{12}$. Suppose $a_{x x} \neq 0$. Then, consider the anticommutator

$$
\left\{T_{x}, \sigma_{x} \otimes \sigma_{x} \otimes 1 \otimes 1\right\} .
$$

On the one hand, this commutes with $P_{Q}$; on the other hand, it equals

$$
a_{x x}\left(1 \otimes \sigma_{x} \otimes \sigma_{x} \otimes 1\right)
$$

thus

$$
1 \otimes \sigma_{x} \otimes \sigma_{x} \otimes 1
$$

commutes with $P_{Q}$. Suppose $a_{y x}$ were also nonzero; then

$$
1 \otimes \sigma_{y} \otimes \sigma_{x} \otimes 1
$$

would also commute with $P_{Q}$, implying that

$$
1 \otimes \sigma_{z} \otimes 1 \otimes 1
$$

commuted with $P_{Q}$, contradicting the fact that $Q$ is pure of minimum distance 2. So at most one of $a_{x x}, a_{y x}$, and $a_{z x}$ is nonzero; since their norms add to 1 , exactly one of them must be nonzero. We can conclude, therefore, that the coefficients $a_{\text {?? }}$ determine a permutation of $\{x, y, z\}$; using the remaining freedom in the third qubit, we may map this permutation to the identity.

Thus we can arrange for $Q$ to commute with

$$
G_{13}=\left\langle\sigma_{x} \otimes 1 \otimes \sigma_{x} \otimes 1, \sigma_{z} \otimes 1 \otimes \sigma_{z} \otimes 1\right\rangle
$$

via transformations in the third qubit only; similarly, $G_{14}$ can be assumed to commute with $Q$. But then $P_{Q}$ must be a linear combination of $1 \otimes 1 \otimes 1 \otimes 1, \sigma_{x} \otimes \sigma_{x} \otimes \sigma_{x} \otimes \sigma_{x}, \sigma_{y} \otimes \sigma_{y} \otimes \sigma_{y} \otimes \sigma_{y}$ and $\sigma_{z} \otimes \sigma_{z} \otimes \sigma_{z} \otimes \sigma_{z}$. This can only happen when $Q$ is a coset of the $[[4,2,2]]$, and is thus equivalent to the $[[4,2,2]]$.

Tracing away any two qubits of a $((6,1,4))$ gives a $((4,4,2))$; this allows us to prove

Corollary 9: Any (pure) $((6,1,4))$ is locally equivalent to quantum hexacode; that is, the $[[6,0,4]]$ with basis

$\begin{array}{cccccc}0 & 0 & 1 & 1 & 1 & 1 \\ 0 & 0 & \omega & \omega & \omega & \omega \\ 1 & 1 & 1 & 1 & 0 & 0 \\ \omega & \omega & \omega & \omega & 0 & 0 \\ 0 & 1 & 0 & 1 & \omega & \bar{\omega} \\ 0 & \omega & 0 & \omega & \bar{\omega} & 1\end{array}$

Proof: Let $v$ be a (pure) $((6,1,4))$. Then for each pair $S$ of qubits

$$
4 \operatorname{Tr}_{S}\left(v v^{\dagger}\right)
$$

is the projection operator onto a $((4,4,2))$, and is thus equivalent to the $[[4,2,2]]$. In particular, we may assume without loss of generality that

$$
4 \operatorname{Tr}_{12}\left(v v^{\dagger}\right)
$$

is given by the $[[4,2,2]]$. In particular, it follows that $v v^{\dagger}$ commutes with

$$
\begin{gathered}
\left\langle 1 \otimes 1 \otimes \sigma_{x} \otimes \sigma_{x} \otimes \sigma_{x} \otimes \sigma_{x},\right. \\
\left.1 \otimes 1 \otimes \sigma_{z} \otimes \sigma_{z} \otimes \sigma_{z} \otimes \sigma_{z}\right\rangle .
\end{gathered}
$$

Then $4 \operatorname{Tr}_{56}\left(v v^{\dagger}\right)$ commutes with

$$
\left\langle 1 \otimes 1 \otimes \sigma_{x} \otimes \sigma_{x}, 1 \otimes 1 \otimes \sigma_{z} \otimes \sigma_{z}\right\rangle .
$$

By the proof of Theorem 8, it follows that we may, by transforming only the first two qubits, assume that $4 \operatorname{Tr}_{56}\left(v v^{\dagger}\right)$ is the $[[4,2,2]]$ as well. Thus $v v^{\dagger}$ must commute with the group

$$
\begin{gathered}
\left\langle 1 \otimes 1 \otimes \sigma_{x} \otimes \sigma_{x} \otimes \sigma_{x} \otimes \sigma_{x},\right. \\
1 \otimes 1 \otimes \sigma_{z} \otimes \sigma_{z} \otimes \sigma_{z} \otimes \sigma_{z}, \\
\sigma_{x} \otimes \sigma_{x} \otimes 1 \otimes 1 \otimes \sigma_{x} \otimes \sigma_{x}, \\
\left.\sigma_{z} \otimes \sigma_{z} \otimes 1 \otimes 1 \otimes \sigma_{z} \otimes \sigma_{z}\right\rangle .
\end{gathered}
$$

Now, consider $P_{13}=4 \operatorname{Tr}_{13}\left(v v^{\dagger}\right)$. This must commute with

$$
\begin{gathered}
\left\langle 1 \otimes \sigma_{x} \otimes \sigma_{x} \otimes \sigma_{x},\right. \\
1 \otimes \sigma_{z} \otimes \sigma_{z} \otimes \sigma_{z}, \\
\sigma_{x} \otimes 1 \otimes \sigma_{x} \otimes \sigma_{x}, \\
\left.\sigma_{z} \otimes 1 \otimes \sigma_{z} \otimes \sigma_{z}\right\rangle .
\end{gathered}
$$

It follows that $P_{13}$ can be written as

$$
\begin{aligned}
& \quad a \cdot 1 \otimes 1 \otimes 1 \otimes 1 \\
& +b \cdot \sigma_{x} \otimes \sigma_{x} \otimes \sigma_{y} \otimes \sigma_{z} \\
& +c \cdot \sigma_{y} \otimes \sigma_{y} \otimes \sigma_{z} \otimes \sigma_{x} \\
& +d \cdot \sigma_{z} \otimes \sigma_{z} \otimes \sigma_{x} \otimes \sigma_{y} \\
& +e \cdot \sigma_{x} \otimes \sigma_{x} \otimes \sigma_{z} \otimes \sigma_{y} \\
& +f \cdot \sigma_{y} \otimes \sigma_{y} \otimes \sigma_{x} \otimes \sigma_{z} \\
& +g \cdot \sigma_{z} \otimes \sigma_{z} \otimes \sigma_{y} \otimes \sigma_{x} .
\end{aligned}
$$

Since $\operatorname{Tr}\left(P_{13}\right)=4$, we must have $a=\frac{1}{4}$. (The remaining nine Pauli operators that commute with (4) have 1 as a component, and thus, since $P_{13}$ is a $((4,4,2))$, cannot appear.) Consider the equation $P_{13}^{2}=P_{13}$. For this to hold, we must, in particular, have that the only terms appearing in the expansion of $P_{13}^{2}$ are the terms appearing in the expansion of $P_{13}$. In particular, consider the term

$$
1 \otimes 1 \otimes \sigma_{x} \otimes \sigma_{x} .
$$

On the one hand, this must have coefficient 0 ; on the other hand, we see that it has coefficient $2 b e$. Thus $b e=0$; without loss of generality, we may assume that $e=0$. It follows that $|b|=\frac{1}{4}$, and

$$
\sigma_{x} \otimes \sigma_{x} \otimes \sigma_{y} \otimes \sigma_{z}
$$

stabilizes $P_{13}$. This implies $f=g=0$ and $|c|=|d|=\frac{1}{4}$. 
But then $v v^{\dagger}$ commutes with the group

$$
\begin{aligned}
& \left\langle 1 \otimes 1 \otimes \sigma_{x} \otimes \sigma_{x} \otimes \sigma_{x} \otimes \sigma_{x},\right. \\
& 1 \otimes 1 \otimes \sigma_{z} \otimes \sigma_{z} \otimes \sigma_{z} \otimes \sigma_{z}, \\
& \sigma_{x} \otimes \sigma_{x} \otimes 1 \otimes 1 \otimes \sigma_{x} \otimes \sigma_{x}, \\
& \sigma_{z} \otimes \sigma_{z} \otimes 1 \otimes 1 \otimes \sigma_{z} \otimes \sigma_{z}, \\
& 1 \otimes \sigma_{x} \otimes 1 \otimes \sigma_{x} \otimes \sigma_{y} \otimes \sigma_{z}, \\
& \left.1 \otimes \sigma_{z} \otimes 1 \otimes \sigma_{z} \otimes \sigma_{x} \otimes \sigma_{y}\right\rangle .
\end{aligned}
$$

It follows immediately that $v$ is equivalent to hexacode.

Corollary 10: Any $((5,2,3))$ is locally equivalent to length 5 Hamming code; that is, the $[[5,1,3]]$ with basis

$\begin{array}{ccccc}0 & 1 & 1 & 1 & 1 \\ 0 & \omega & \omega & \omega & \omega \\ 1 & 0 & 1 & \omega & \bar{\omega} \\ \omega & 0 & \omega & \bar{\omega} & 1\end{array}$

Proof: Let $Q$ be the given $((5,2,3))$. There exists a self-dual code $Q^{\prime}$ of length 6 such that $2 \operatorname{Tr}_{1}\left(P_{Q^{\prime}}\right)=P_{Q}$ [7, Theorem 21]. But then we can compute the weight enumerator of $Q^{\prime}$; this tells us that $Q^{\prime}$ is a $((6,1,4))$, and thus locally equivalent to hexacode. But then the code obtained by tracing away the first qubit of hexacode must be locally equivalent to $Q$; in other words, $Q$ is locally equivalent to the $[[5,1,3]]$.

The proof of Theorem 8 can be used to show that any $\left(\left(2 m, 4^{m-1}, 2\right)\right)$ with the same quartic invariants as the $[[2 m, 2 m-$ $2,2]]$ is locally equivalent to the $[[2 m, 2 m-2,2]]$. This suggests that it may be possible to give some set of conditions on quartic invariants, satisfied by all additive codes, such that any code satisfying the conditions is equivalent to an additive code. Note that this cannot be true of cubic invariants, since the codes of Theorem 7 have the same cubic invariants as the additive codes with the same parameters [5, Lemma 5].

\section{AUTOMORPHISMS AND EQUIVALENCES}

In [1], the automorphism group of an additive code is defined to be the subgroup of the natural semidirect product of $S_{n}$ and $S_{3}^{n}$ that preserves the code. There is also a natural concept of automorphism group for general codes, to wit, the group of all equivalences from the code to itself; we will call this the full automorphism group. The full automorphism group is of particular interest because automorphisms of a code induce fault-tolerant operations on the encoded state [2]. It is natural, therefore, to wonder how these two concepts of automorphism groups are related.

Recall that the "extraspecial group" $E$ is the group generated by $i I$ as well as all tensor products of Pauli matrices. The Clifford group is then the subgroup of the normalizer of $E$ with coefficients from $\mathbb{Q}[(1+i) / \sqrt{2}]$. See [1], [10] for more information.

Theorem 11: Let $Q$ be an additive code, with full automorphism group $A$, and with automorphism group $A_{0}$ as an additive code. Then the intersection of $A$ with the Clifford group is isomorphic to the semidirect product of $A_{0}$ and the centralizer of $Q$ in the extraspecial group.

Proof: We can map $S_{3}$ into $S O(3)$ by

$$
\begin{aligned}
&(12) \mapsto\left(\begin{array}{rrr}
0 & -1 & 0 \\
-1 & 0 & 0 \\
0 & 0 & -1
\end{array}\right) \\
&(23) \mapsto\left(\begin{array}{rrr}
-1 & 0 & 0 \\
0 & 0 & -1 \\
0 & -1 & 0
\end{array}\right) .
\end{aligned}
$$

This induces, in the usual way, a map from $S_{3}$ to $P S U(2)$, and thus a map from $A_{0}$ to $P S U(2)^{\otimes n}$. Let $\phi$ be any equivalence in the image of this map. We readily verify that the centralizer of $\phi(Q)$ in the extraspecial group $E$ is isomorphic to the centralizer of $Q$ in $E$; consequently, there exists some element $e \in E$ such that $e \phi(Q)=Q$. On the other hand, if we reduce the intersection of $A$ with the Clifford group modulo $E$, then we must get $A_{0}$; the result follows immediately.

The natural question is then: When are these groups isomorphic? Consideration of the $[[2,0,2]]$ reveals that it is possible for an additive code to have nonadditive automorphisms.

Lemma 12: The full automorphism group of the $((2,1,2))$ is isomorphic to the semidirect product of $Z_{2}$ and $\operatorname{PSU}(2)$, with $Z_{2}$ acting on $P S U(2)$ as complex conjugation.

Proof: By Lemma 5, we may assume that the code is $|00\rangle+|11\rangle$. As in Lemma 5, we may reduce the problem to one of unitary matrices; we immediately find that any local equivalence must be of the form $U \otimes \bar{U}$ for $U \in P S U(2)$. The equivalence which exchanges the two qubits completes the group.

On the other hand, the larger [[2m,2m-2,2]]'s behave much better.

Theorem 13: Let $Q$ be a [[2m, $2 m-2,2]]$ for $m \geq 2$. Then every automorphism of $Q$ lies in the Clifford group.

Proof: Since the additive automorphism group of $Q$ acts transitively on the qubits, it suffices to consider local automorphisms. In particular, any local equivalence corresponds to an $2 m$-tuple of elements of $S O(3)$; we need to show that every element of the $2 m$-tuple is a monomial matrix. Without loss of generality, we may assume that $Q$ is given by the projection operator

$$
P_{Q}=\frac{1}{4}\left(I^{\otimes 2 m}+\sigma_{x}^{\otimes 2 m}+(-1)^{m} \sigma_{y}^{\otimes 2 m}+\sigma_{z}^{\otimes 2 m}\right) .
$$

The key observation is to note that

$$
4 P_{Q}-I^{\otimes 2 m}
$$

is naturally associated to the following vector in $\left(\mathbb{R}^{3}\right)^{\otimes 2 m}$

$$
v=|000 \cdots\rangle+(-1)^{m}|111 \cdots\rangle+|222 \cdots\rangle
$$

acted on by $S O(3)^{\otimes 2 m}$ in the natural way. So the question is then: for which elements of $S O(3)^{\otimes 2 m}$ is $v$ a fixed point?

Consider the operator

$$
\left\langle\left. 0\right|_{1} \operatorname{Tr}_{\{3,4 \cdots 2 m\}}\left(v v^{t}\right) \mid 0\right\rangle_{1}
$$

acting on the second "trit" (that is, copy of $\mathbb{R}^{3}$ ). We readily see that this is proportional to $|0\rangle\langle 0|$; in particular, it has rank 1 . Consequently, if $\phi \in S O(3)^{\otimes 2 m}$ admits $v$ as a fixed point, then the operator

$$
\left\langle\left. 0\right|_{1} \operatorname{Tr}_{\{3,4 \cdots 2 m\}}\left(\phi(v) \phi(v)^{t}\right) \mid 0\right\rangle_{1}
$$

must have rank 1. Clearly, this depends only on the action of $\phi$ on the first trit. Thus the condition must still be satisfied if we replace $\phi$ by

$$
\phi^{\prime}=\phi^{(1)} \otimes I^{\otimes 2 m-1} \text {. }
$$

Then we can readily compute

$$
\left\langle\left. 0\right|_{1} \operatorname{Tr}_{\{3,4 \cdots 2 m\}}\left(\phi^{\prime}(v) \phi^{\prime}(v)^{t}\right) \mid 0\right\rangle_{1}
$$

by first noting that

$$
\begin{aligned}
\operatorname{Tr}_{\{3,4, \cdots 2 m\}}\left(\phi^{\prime}(v) \phi^{\prime}(v)^{t}\right) \\
\quad=\left(\phi^{(1)} \otimes I\right) \operatorname{Tr}_{\{3,4, \cdots 2 m\}}\left(v v^{t}\right)\left(\phi^{(1)} \otimes I\right)^{t} \\
\propto\left(\phi^{(1)} \otimes I\right)(|00\rangle\langle 00|+| 11\rangle\langle 11|+| 22\rangle\langle 22|)\left(\phi^{(1)} \otimes I\right)^{t}
\end{aligned}
$$


since $m \geq 2$. Selecting out the submatrix in which the first trit is 0 , we get

$$
\left(\begin{array}{ccc}
\left(\phi_{00}^{(1)}\right)^{2} & 0 & 0 \\
0 & \left(\phi_{10}^{(1)}\right)^{2} & 0 \\
0 & 0 & \left(\phi_{20}^{(1)}\right)^{2}
\end{array}\right)
$$

which has rank 1 if and only if exactly one of $\phi_{00}^{(1)}, \phi_{10}^{(1)}$, or $\phi_{20}^{(1)}$ is nonzero. But this must then be true for the other rows of $\phi^{(1)}$; it follows that $\phi^{(1)}$ is a monomial matrix. The theorem follows immediately.

Corollary 14: Any equivalence of GF (4)-linear quantum codes lies in the Clifford group, unless the codes have minimum distance 1 , or contain a codeword of weight 2 .

Proof: It suffices to prove the result for local equivalence, since permuting the qubits of a GF (4)-linear code gives another GF (4)linear code.

Let $C$ be a w.s.d. GF (4)-linear code. A subset $S$ of $\{1,2,3 \cdots n\}$ will be called a minimal support of $C$ if there exists a codeword in $C$ of support $S$, but no nontrivial codewords of support strictly contained in $S$. Similarly, we say a codeword of $C$ is minimal if its support is minimal.

Lemma 15: A GF (4)-linear code $C$ is spanned by its minimal codewords.

Proof: Let $v$ be a codeword with support $S$; we need to show that $v$ is a linear combination of minimal codewords. Either $S$ is minimal, and we are done, or there exists a nontrivial codeword $v_{0}$ with support $S_{0}$ contained in $S$. Let $e$ be any column in $S_{0}$, and consider $v_{1}=v-\left((v)_{e} /\left(v_{0}\right)_{e}\right) v_{0}$. The support of $v_{1}$ does not contain $e$, but is still contained in $S$. The lemma follows by induction.

Remark: A referee has pointed out that this lemma is true for linear codes over arbitrary fields (but not over rings, in general).

For the associated quantum code $Q$, we first note that the minimal supports of $Q$ can be determined from the local weight enumerator of $Q$; consequently, the set of minimal supports is a local invariant. Moreover, if we associate a new code $Q_{S}$ to each minimal support, by selecting out those codewords of $C$ with support $S$, the code $Q_{S}$ can be determined without reference to the additive structure of $Q$. Finally, we note that each $Q_{S}$ is a [[2m,2m-2,2]] for some $m$.

In particular, then, if $Q$ and $Q^{\prime}$ are equivalent additive codes, then they must have the same minimal supports, and for each minimal support, the equivalence must take $Q_{S}$ to $Q_{S}^{\prime}$. From the hypotheses and linearity, it follows that every minimal support has size $2 m$ for $m \geq 2$. From the lemma, we know that $Q$ is the intersection of all the $Q_{S}$ 's; it follows that every qubit is covered by some minimal support. But then it follows from Theorem 13 that the equivalence lies in the Clifford group.

Remark: The hypotheses of the corollary are quite weak; if $Q$ has minimum distance 1 , then some qubit of $Q$ is completely unencoded, and can thus be removed, while if $Q$ has a codeword of weight 2, then $Q$ is the tensor product of a smaller code and a $((2,1,2))$. In both cases, the extra freedom afforded is easy to determine. It should be possible to extend this result to additive codes, under similarly weak hypotheses.

Corollary 16: If $Q$ is a GF (4)-linear code, then every automorphism of $Q$ lies in the Clifford group, unless $Q$ has minimum distance 1 , or contains a codeword of weight 2 .

Proof: This follows immediately from Corollary 14.

In some cases, we can apply Theorem 13 to nonlinear codes.
Corollary 17: Any automorphism of the $[[12,0,6]]$ is contained in the Clifford group.

Proof: The proof of Corollary 16 holds, except that we consider only those minimal supports such that three codewords have that support; there are six such supports that together cover the 12 qubits.

Corollary 18: Let $Q$ be the $((5,6,2))$ defined in [8]. Then every automorphism of $Q$ is contained in the Clifford group; in particular, its full automorphism group is the group of order 3840 given in [8].

Proof: Recall from the remark following Theorem 3 that, for any $((5,6,2))$

$$
I-2 \operatorname{Tr}_{\{1\}}\left(P_{Q}\right)
$$

is the projection operator of a $((4,4,2))$; in the case of the given $((5,6,2))$, the five $((4,4,2))$ 's are all explicitly additive. The result follows easily from Theorem 13 .

\section{ACKNOWLEDGMENT}

The author would like to thank A. R. Calderbank, P. Shor, and $\mathrm{N}$. Sloane for many helpful conversations, as well as the anonymous referees for helpful comments.

\section{REFERENCES}

[1] A. R. Calderbank, E. M. Rains, P. W. Shor, and N. J. A. Sloane, "Quantum error correction via codes over GF (4)," IEEE Trans. Inform. Theory, vol. 44, pp. 1369-1387, July 1998.

[2] D. Gottesman, "A theory of fault-tolerant quantum computation," Phys. Rev. A, vol. 57, pp. 127-156, 1998.

[3] E. Knill and R. Laflamme, "A theory of quantum error correcting codes," Phys. Rev. A, vol. 55, pp. 900-911, 1997.

[4] E. M. Rains, "Nonbinary quantum codes," LANL e-print quant$\mathrm{ph} / 9703048$

[5] _ "Polynomial invariants of quantum codes," LANL e-print quant$\mathrm{ph} / 9704042$.

[6] _Luantum shadow enumerators," LANL e-print quant$\mathrm{ph} / 9611001$.

[7] , "Quantum weight enumerators," IEEE Trans. Inform. Theory, vol 44, pp. 1388-1394, July 1998

[8] E. M. Rains, R. H. Hardin, P. W. Shor, and N. J. A. Sloane, "A nonadditive quantum code," Phys. Rev. Lett., vol. 79, pp. 953-954, 1997.

[9] P. W. Shor and R. Laflamme, "Quantum analog of the MacWilliams identities in classical coding theory," Phys. Rev. Lett., vol. 78, pp. 1600-1602, 1997.

[10] G. E. Wall, "On Clifford collineation, transofrm and similarity groups IV," Nagoya Math. J., vol. 21, pp. 199-222, 1962. 